\title{
1 Coordination of pupil and saccade responses by the superior colliculus
}

3 Chin-An Wang ${ }^{1,2,3,4}$ and Douglas P. Munoz ${ }^{1}$

$5 \quad{ }^{1}$ Centre for Neuroscience Studies, Queen's University, Kingston, Ontario, Canada

$6 \quad{ }^{2}$ Graduate Institute of Mind, Brain, and Consciousness, Taipei Medical University, Taipei,

7 Taiwan

$8{ }^{3}$ Research Center of Brain and Consciousness, Shuang Ho Hospital-Taipei Medical University,

9 Taipei, Taiwan

$10{ }^{4}$ Department of Anesthesiology, Shuang Ho Hospital, Taipei Medical University, Taipei, Taiwan

12 Running head: Superior colliculus coordinates saccades and pupil dilation

14 Keywords: pupil dilation, coordination, orienting, trial-by-trial correlation, background

15 luminance

1736 pages; 5 figures; abstract: 155 words

19 Correspondence:

20 Chin-An Wang

21 Graduate Institute of Mind, Brain, and Consciousness, Taipei Medical University, Taipei,

22 Taiwan. E-mail: josh.wang@tmu.edu.tw

24 Douglas P. Munoz

25 Centre for Neuroscience Studies, Queen's University, Kingston, ON, Canada. E-mail:

26 doug.munoz@queensu.ca

\section{Acknowledgement}

29 This work was supported by Canadian Institutes of Health Research Grant (MOP-FDN-148418)

30 and the Canada Research Chair Program to DPM, and grants from Taiwan Ministry of Science

31 and Technology (108-2410-H-038-002-MY3; 109-2636-H-038-005) to CW. We thank Ann

32 Lablans, Brittney Armitage-Brown, and Mike Lewis for outstanding technical assistance. 


\section{Abstract}

35 The appearance of a salient stimulus evokes saccadic eye movements and pupil dilation as part

36 of the orienting response. Although the role of the superior colliculus (SC) in saccade and pupil

37 dilation has been established separately, whether and how these responses are coordinated

38 remains unknown. The SC also receives global luminance signals from the retina, but whether

39 global luminance modulates saccade and pupil responses coordinated by the SC remains

40 unknown. Here, we used microstimulation to causally determine how the SC coordinates saccade

41 and pupil responses, and whether global luminance modulates these responses by varying

42 stimulation frequency and global luminance in male monkeys. Stimulation frequency modulated

43 saccade and pupil responses, with trial-by-trial correlations between the two responses. Global

44 luminance only modulated pupil, but not, saccade responses. Our results demonstrate an

45 integrated role of the SC on coordinating saccade and pupil responses, characterizing luminance

46 independent modulation in the SC, together elucidating the differentiated pathways underlying

47 this behavior. 


\section{Introduction}

A series of responses, including saccadic eye movements and pupil dilation, are evoked by the presentation of a salient stimulus as part of the orienting response ${ }^{1-3}$. Recent evidence,

51 particularly in monkeys, has shown that pupil dilation is evoked by presentation of a visual

52 target, enhanced by acoustic stimuli, modulated by stimulus saliency, and triggered by

53 microstimulation of the superior colliculus (SC) ${ }^{4-7}$, a midbrain sensorimotor center that is

54 phylogenetically conserved and causally involved in producing orienting responses ${ }^{8-12}$. Pupil

55 size and saccadic eye movements are becoming popular and promising indices of cognitive and

56 disease processes ${ }^{13}$. Are the neural mechanisms underlying the cognitive modulation for pupil

57 size similar to those for saccades? Further, what is the role of the SC in coordinating saccade and

58 pupil responses?

The SC is organized into a retinotopic map of contralateral visual space and has

anatomically and functionally differentiated superficial (SCs) and intermediate/deep (SCi) layers

$61{ }^{14}$. The SCs receives visual signals from the retina and visual cortex, whereas the SCi receives

62 multisensory and cognitive inputs from various cortical and subcortical areas ${ }^{15-18}$, including

63 structures and neuromodulatory systems involved in the control of pupil size, such as the locus

64 coeruleus (LC), frontal eye field (FEF), and cholinergic projections from the pedunculopontine

65 tegmental nucleus (PPTN) ${ }^{19-23}$. Moreover, the SCi projects to the premotor circuitry in the

66 brainstem that initiates the orienting response ${ }^{8,10,24}$. Electrical microstimulation of the SCi in

67 monkeys induces saccades ${ }^{25}$, and the properties of the evoked movements are modulated by

68 stimulation parameters and the location of stimulation within the SC, with larger saccade

69 responses (e.g., peak velocity) evoked using stronger stimulation parameters and larger

70 amplitude saccades evoked by moving toward the caudal $\mathrm{SCi}^{26}$. Weak microstimulation in the 
71 SCi can evoke pupil dilation without inducing saccades ${ }^{4,22}$. If the saccade and pupil responses

72 are coordinated by the SCi (referred to as the common drive hypothesis), then varying

73 stimulation parameters and changing stimulation sites should modulate not only saccades, but

74 also pupil responses.

75 Besides orienting, pupil size also changes to regulate the amount of light entering the retina

76 to optimize visual sensitivity and acuity ${ }^{27,28}$, with smaller pupil sizes for higher luminance

77 levels, and larger pupil size for lower luminance levels. This luminance response is controlled by

78 the balance of activity between the parasympathetic and sympathetic nervous systems ${ }^{29,30}$.

79 Specifically, higher parasympathetic activity occurs during higher luminance levels causing

80 pupil constriction, while higher sympathetic activity occurs during lower luminance levels

81 leading to pupil dilation. The SCs receives direct retinal projections including those from

82 intrinsically photosensitive retinal ganglion cells (ipRGCs) ${ }^{31}$, which are important for luminance

83 encoding and regulating the pupil light reflex ${ }^{32-35}$. Although visual signals such as visual

84 contrast play an important role in the $\mathrm{SC}^{36}$, the functional role of global luminance signals in the

85 SC is yet to be explored.

86 Using microstimulation of the SCi with a systematic variation of stimulation frequency and

87 background luminance, we investigate the role of the SCi in coordinating saccade and pupil

88 responses at different luminance levels. If the SCi coordinates saccades and pupil responses, then

89 responses evoked by SCi microstimulation should be correlated on trial-by-trial basis.

90 Additionally, if the SCi is modulated by luminance levels, then background luminance should

91 affect both pupil and saccade responses evoked by SCi microstimulation (Fig 1A). If, by

92 contrast, the luminance signals sent to the SCi are functionally insignificant, then only the

93 evoked pupil responses will be modulated ${ }^{29}$. 

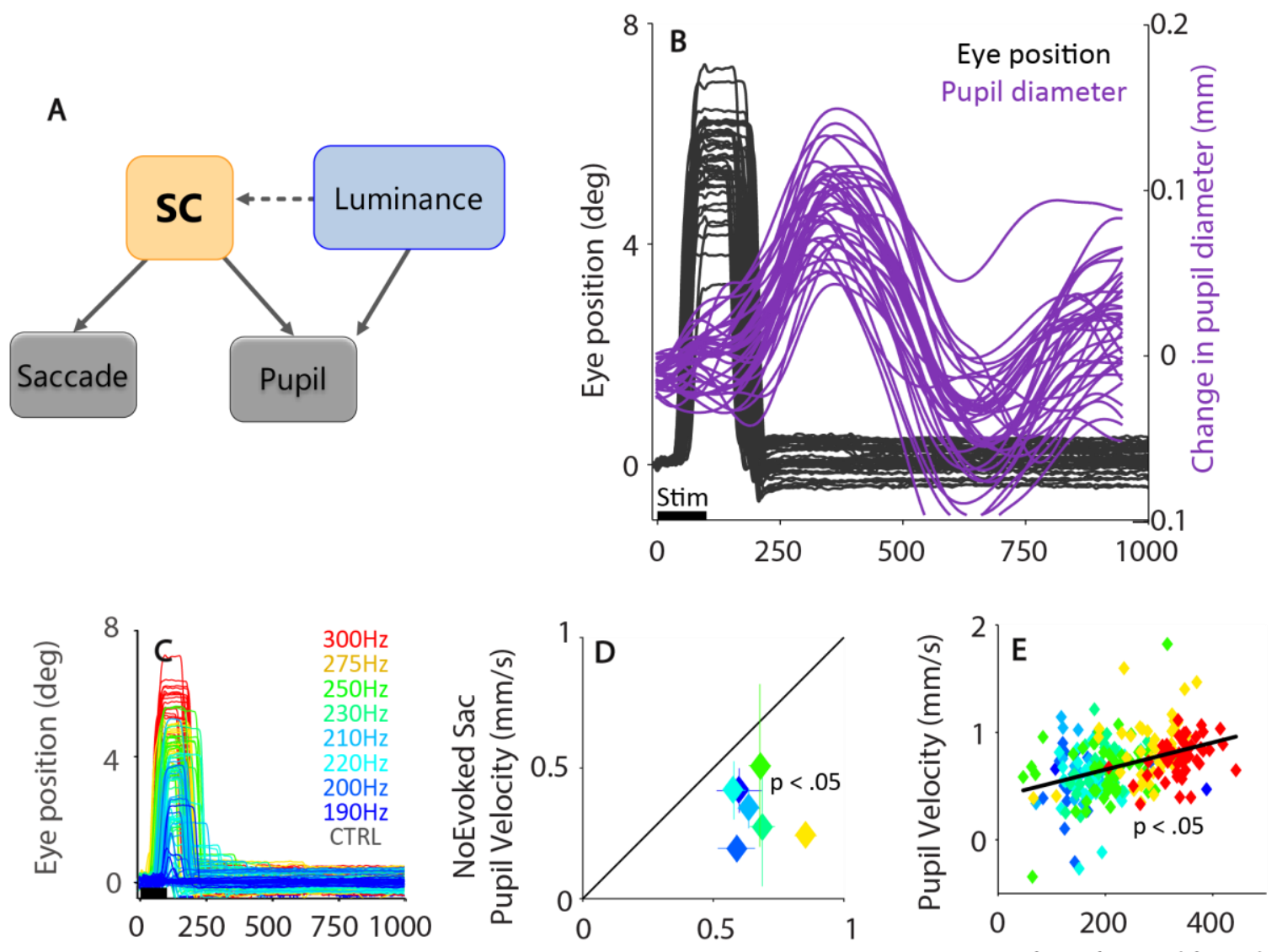

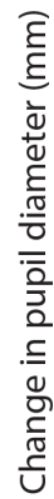

Pupil velocity $(\mathrm{mm} / \mathrm{s})$

Saccade Velocity (deg/s)
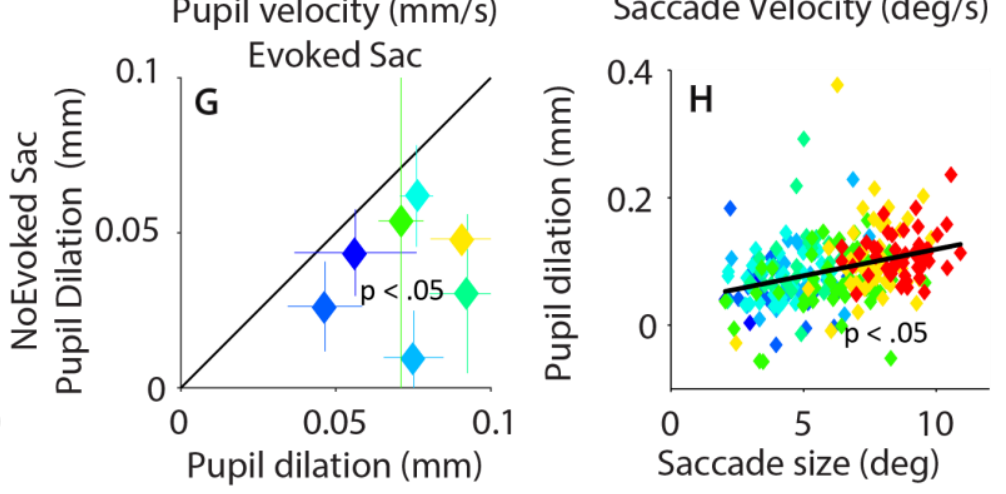

Figure 1. Effect of SCi microstimulation on saccade and pupil responses from an

example site. (A) Illustration of hypotheses on the modulation of the superior colliculus

on saccade and pupil responses by luminance. (B) Temporal dissociation between evoked saccades (only horizontal eye position) and pupil responses. (C,F) Effects of stimulation frequency on (C) saccade and (F) pupil responses. (D,G) Pupil responses between trials with evoked saccades and trials without evoked saccades in (D) velocity and (G) 
magnitude. (E,H) Trial-by-trial correlation between normalized pupil and saccade responses in $(\mathrm{E})$ velocity and $(\mathrm{H})$ magnitude. In C,F, the black bar on X-axis indicates the time line of microstimulation. The shaded colored regions surrounding the pupil response represent \pm standard error range in different conditions. CTRL: control (no stimulation).

\section{Results} Stim: microstimulation.

\section{Correlation between saccade and pupil responses via varying train frequency}

If the mechanisms of initiation of saccade and pupil dilation are shared through the $\mathrm{SCi}$, then

111 should be observed. More specifically, the common-drive hypothesis postulates that the same

112 efferent neurons in the SCi project to both saccade and pupil control circuits. Therefore, trial-by-

113 trial correlations between saccade and pupil responses induced by SCi microstimulation should

114 be observed. For example: if there are stronger output signals from the SCi, this signal should

115 drive larger saccade and pupil responses. Moreover, because of a gating role of the omnipause

116 neurons e.g., ${ }^{37}$, signals must surpass the threshold to evoke saccades. Thus, pupil dilation on

117 trials with an evoked saccade should be larger than that on trials without an evoked saccade. To

118 test these predictions, monkeys were trained to perform a simple fixation task in which they were

119 required to maintain fixation upon a central fixation point (FP). Microstimulation was delivered

120 to 24 sites in the intermediate layers ( $\mathrm{SCi}$ ) (9 in monkey A, 15 in monkey $\mathrm{B}$ ) on $50 \%$ of trials

121 (see Materials and Methods for details). Figure 1B shows an example of individual saccade and

122 pupil responses induced by SCi microstimulation $(300 \mathrm{~Hz}, 40 \mu \mathrm{A}, 100 \mathrm{~ms})$. Microstimulation

123 evoked saccades (black traces in Fig. 1B) towards the location that corresponds to the stimulated 
124 SC position with a mean latency of $42 \mathrm{~ms}(22-63 \mathrm{~ms})$ after stimulation onset. The monkey then

125 quickly returned his eyes to the central FP with a mean latency of $125 \mathrm{~ms}$ (87 - $152 \mathrm{~ms})$

126 measured after the previous saccade offset. Pupil dilation was also evoked after

127 microstimulation, but the pupil response onset latency was slower, starting near $200 \mathrm{~ms}$ and

128 peaking near $300 \mathrm{~ms}$ after the stimulation, due to the longer efferent delay of the pupil control

129 circuit $^{4}$. We focused here on initial pupil dilation because pupil dilation has long been

130 characterized as a component of orienting ${ }^{1,38}$. Moreover, pupil dilation dynamics evoked by SCi

131 microstimulation ${ }^{4}$ are similar to those evoked by salient stimuli ${ }^{6}$, suggesting that similar

132 orienting processes may be involved between two observed pupil dilation. Also, pupil dilation is

133 related to aspects of cognition ${ }^{39,40}$. Eye movements and variations in eye position could diminish

134 the accuracy of pupil size measurements. Because the eyes of monkeys almost always returned

135 to central FP within $300 \mathrm{~ms}$ after stimulation onset (Fig. 1B), to maintain the accuracy of pupil

136 measurement, we measured pupil peak velocity and dilation (which occurred at around 300 to

$137500 \mathrm{~ms}$ after stimulation onset) to correlate pupil responses with saccade responses (see

138 Materials and Methods).

139 Changes in stimulation frequency altered saccade and pupil responses in a systematic and

140 coordinated manner, as shown in Fig. 1C-H for this example stimulation site. Consistent with the

141 literature ${ }^{26}$, increasing stimulation frequency systematically increased the probability of evoking

142 a saccade (low to high frequency: 13, 33, 48, 64, 81, 92, 98, $100 \%$ ), decreased the latency (low

143 to high frequency, mean value: $86.3,72.1,69.3,78.2,67.2,54.0,41.7,41.8 \mathrm{~ms}$ ), and increased

144 saccade amplitude (Fig. 1C, low to high frequency conditions: 3.8, 4.1, 4.6, 4.2, 4.7, 6.1, 7.6, 8.7

$145 \mathrm{deg}$ ). Microstimulation also resulted in transient pupil dilation, followed by constriction (purple

146 traces in Fig. 1B). Importantly, these pupil responses were also systematically modulated by 
147 stimulation frequency, with larger peak dilation observed for higher stimulation frequencies (Fig.

$1481 \mathrm{~F}$, low to high frequency in magnitude: $0.048,0.052,0.080,0.073,0.099,0.072,0.105,0.109$

$149 \mathrm{~mm})$.

150 To examine the trial-by-trial correlation between saccade and pupil responses, we divided 151 pupil responses according to the likelihood of evoking saccades. Trials with saccades were 152 associated with larger pupil velocities and greater dilation (Fig. 1D velocity, $t(6)=5.05, p<$ 1530.01 ; Fig. $1 \mathrm{G}$ magnitude, $t(6)=3.86, p<0.01$ ). We also examined the correlation between 154 saccade and pupil responses across individual trials when a saccade was evoked and found that 155 trials with larger saccade responses had larger pupil responses (Fig. $1 \mathrm{E}$ in velocity, $\mathrm{R}=0.4, p<$ 156 0.001; Fig. 1H in magnitude, $\mathrm{R}=0.34, p<0.001)$.

157 Saccade and pupil responses evoked by microstimulation were highly correlated across our 158 sample of stimulation sites $(\mathrm{N}=24)$ in two monkeys (Fig. 2). Stimulation frequency 159 systematically modulated the probability of evoking a saccade (Fig. 2A). A generalized linear 160 model was used to characterize this relationship by applying a binomial distribution (saccade 161 evoked or not: 1 or 0 ) and a logistic link function (a logistic fit). The fitted model yielded an R162 squared value of 0.99 (data from data source Fig. 2), suggesting that saccade probability can 163 almost be fully explained by the model of using stimulation frequency as a predictor. Fig. 2B

164 summarizes pupil dynamics following microstimulation (22 sites for which we had four or more 165 stimulation frequency conditions were included in Fig. 2B, 2 sites that only had three stimulation 166 frequency conditions were thus excluded from analysis, $\mathrm{N}=22$; individual monkey data provided 167 in Supplementary Figure 1, revealing the same stimulation frequency modulation in two 168 monkeys), showing that pupil dilation scaled with stimulation frequency (high to low frequency 169 and control: mean pupil size at an epoch of 250 to $350 \mathrm{~ms}$ after stimulation onset: $0.075,0.053$, 
$1700.041,0.021,-0.006 \mathrm{~mm})$. To illustrate the results across microstimulation sites, we normalized

171 the data by dividing saccade/pupil values by the median value in the highest frequency condition

172 (see Materials and Methods). The common drive hypothesis (Fig. 1A) predicts that pupil

173 responses should be larger on trials in which saccades were evoked, and this is what we

174 observed. Pupil responses evoked by microstimulation were larger on trials with saccades,

175 compared to trials without saccades (Fig. $2 \mathrm{C}$ in velocity, $t(47)=3.4, p<0.01$; Fig. 2D in

176 magnitude, $t(47)=2.55, p<0.05)$. Saccade and pupil responses were both systematically

177 influenced by stimulation frequency (velocity: Sac: Fig. 2E, R = 0.75, Pupil: Fig. 2F, R = 0.57;

178 magnitude: Sac: Fig. 2I, $\mathrm{R}=0.69$, Pupil: Fig. 2J, $\mathrm{R}=0.61$, all $p<0.001)$. Consistent with the

179 common drive hypothesis, trial-by-trial correlational analyses collapsed across all frequency

180 conditions from each stimulation site $(\mathrm{N}=24)$ showed a reliable positive correlation between

181 pupil and saccade responses (histograms of the correlation coefficients are shown in Fig. 2G, K),

182 and the mean correlation coefficient was 0.36 in velocity with significant correlations $(p<.05)$

183 obtained for 58\% (14/24) of the stimulation sites (Fig. 2G, parried t-test of $R$-values against

184 zeros: $t(23)=7.6, p<0.001)$, and 0.29 in magnitude with significant correlations obtained for

$18567 \%(16 / 24)$ of the stimulation sites (Fig. $2 \mathrm{~K}, t(23)=7.1, p<0.001)$. Again, these results were

186 not an artifact of inaccurate measurement of pupil size because pupil responses relative to the

187 return saccade onset were clearly modulated by stimulation frequency with larger dilation

188 observed for higher stimulation frequencies (Supplementary Figure 2). 

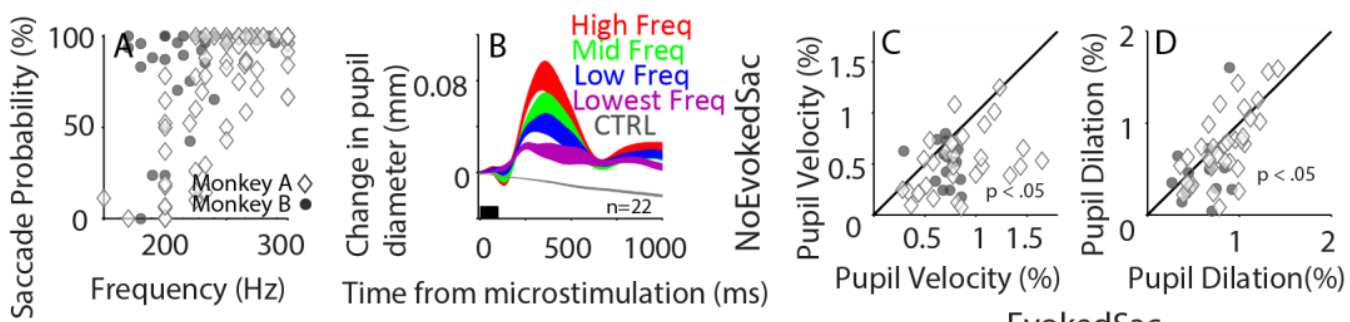

EvokedSac
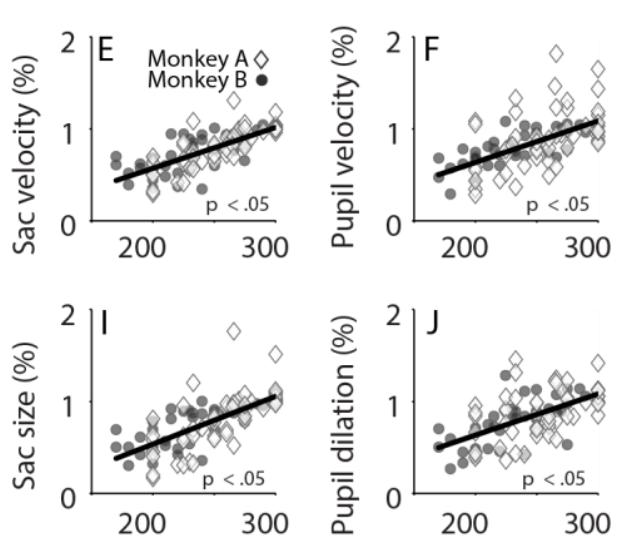

Frequency $(\mathrm{Hz})$

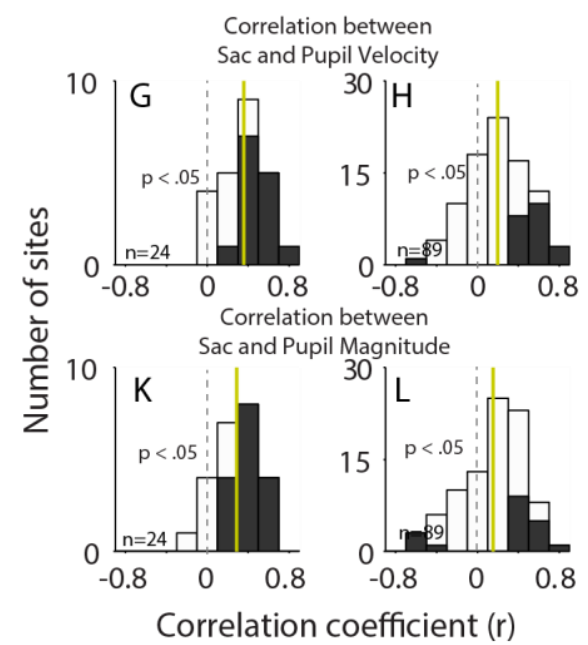

Correlation coefficient $(r)$ responses after microstimulation in four highest frequency and control (no microstimulation) conditions on data collapsed across monkeys and stimulation sites $(n=22)$. (C,D) Pupil responses collapsed across monkeys and stimulation sites $(n=24)$ between trials with evoked saccades and trials without evoked saccades in $(\mathbf{C})$ velocity and (D) magnitude. (E,F,I,J) Effect of stimulation frequency on evoked saccade and coefficients for the relationship between evoked saccade and pupil responses in $(\mathbf{G})$ velocity and $(\mathbf{K})$ magnitude $(n=24)$. $(\mathbf{H}, \mathbf{L})$ Distribution of correlation coefficients for the pupil responses in $(\mathbf{E}, \mathbf{F})$ velocity and $(\mathbf{I}, \mathbf{J})$ magnitude. $(\mathbf{G}, \mathbf{K})$ Distribution of correlation relationship between evoked saccade and pupil responses in each frequency condition in velocity (H) and magnitude (I) $(\mathrm{n}=89)$. In $\mathbf{A}, \mathbf{E}, \mathbf{F}, \mathbf{I}, \mathbf{J}$, Black lines indicate the regression line. In $\mathbf{B}$, the black bar on $\mathrm{X}$-axis indicates the time line of microstimulation. The shaded 
colored regions surrounding the pupil response represent \pm standard error range for different conditions. In $\mathbf{G}, \mathbf{H}, \mathbf{K}, \mathbf{L}$, the vertical black dotted and yellow solid line represent a zero and median value of correlation coefficient. Filled-bars indicate sites with statistically significant correlation $(p<0.05)$. n: number of sites. CTRL: control (no stimulation).

To further examine the correlation between saccade and pupil responses from individual

210 frequency conditions at each site, we split trials according to median saccade peak velocity or

211 magnitude. Although the variation across trials was reduced in a given frequency condition, trials

212 with larger saccade responses still had larger pupil responses (large saccade velocity: $0.85 \mathrm{~mm} / \mathrm{s}$

213 pupil velocity; small saccade velocity: $0.73 \mathrm{~mm} / \mathrm{s}$ pupil velocity; $t(88)=4.94, p<0.001$;

214 magnitude: large saccade size: 0.095 mm diameter; small saccade size: 0.077 mm diameter; $t(88)$

$215=5.58, p<0.001)$. Figure $2 \mathrm{H}$ and $\mathrm{L}$ show summary histograms of correlation coefficients for all

216 conditions, revealing in general positive correlations between saccade and pupil responses in

217 velocity (significant positive correlations for $24 \%(21 / 89)$ of the individual

218 stimulation/conditions; mean correlation coefficient of $0.2, t(88)=6.5, p<0.001)$ and in

219 magnitude (significant positive correlations for 17\% (15/89) of the stimulation/conditions; mean

220 correlation coefficient of $0.15, t(88)=4.6, p<0.001$ ), though there were a few sites having

221 significant negative correlations (velocity: velocity: 1\%, 1/89; magnitude: 4\%, 4/89). Together,

222 these results demonstrate positive trial-by-trial correlations between saccade and pupil responses

223 evoked by the SCi microstimulation. Similar results were obtained with varying stimulation

224 duration (Supplementary Figure 3). 

sample of stimulation sites in two monkeys (data from Fig. 2E,F,I,J) using stimulation frequency (F) and saccade responses (S) as independent variables in the analysis.

$$
\text { Pupil dilation }=\beta \_1 * \mathrm{~F}+\beta \_2 * \mathrm{~S}+\beta \_0
$$

$234 \quad \beta \_0, \beta \_1, \beta \_2$ were constant linear weights generated by the model.

235 The results yielded $\beta \_1=0.0007, p=0.40 ; \beta \_2=0.8475, p=1.5 \mathrm{e}-7 ; \mathrm{R}=0.71$ in the velocity analysis, and $\beta \_1=0.0017, p=0.025 ; \beta \_2=0.5431, p=2.1 \mathrm{e}-7 ; \mathrm{R}=0.73$ in the magnitude analysis, suggesting that pupil responses were significantly predicted by the saccade response in

238 both velocity and magnitude analyses, but only significantly predicted by stimulation frequency

239 in magnitude analysis, These results imply that the pupil response is more reliably accounted by

240 the saccade response.

242 Site-specific effects in saccade and pupil responses

243 The SCi is organized into a retinotopically coded motor map, and different sites evoke

244 different amplitude and direction saccades ${ }^{25}$ because different sites project disproportionally to

245 the omnipause and burst neurons ${ }^{41-43}$. Although unidentified, this pattern of anatomical

246 relationship may also be present in the $\mathrm{SCi}$ projections to the pupil control circuit; that is, regions

247 in the SCi map that code for larger amplitude saccades (i.e., caudal SC) may also code for larger 
pupil dilation, yielding more and/or stronger projections from the caudal SC to the pupil control circuit. Because pupil size is unidimensional, we used eccentricity of the response field determined by the vector of saccade elicited with suprathreshold SCi stimulation (see Materials and Methods) to investigate the relationship between pupil dilation magnitude and evoked saccade size. The magnitude of the evoked pupil dilations was correlated with RF eccentricity: larger dilation and faster velocities were indeed observed for sites with larger vectors of evoked saccades (Fig. 3A in magnitude: $\mathrm{R}=0.73, p<.001$; and 3B in velocity: $\mathrm{R}=0.78, p<.001$ ). Therefore, our microstimulation results demonstrate the site-specific modulation of both saccade and pupil responses, which is consistent with the common drive hypothesis.
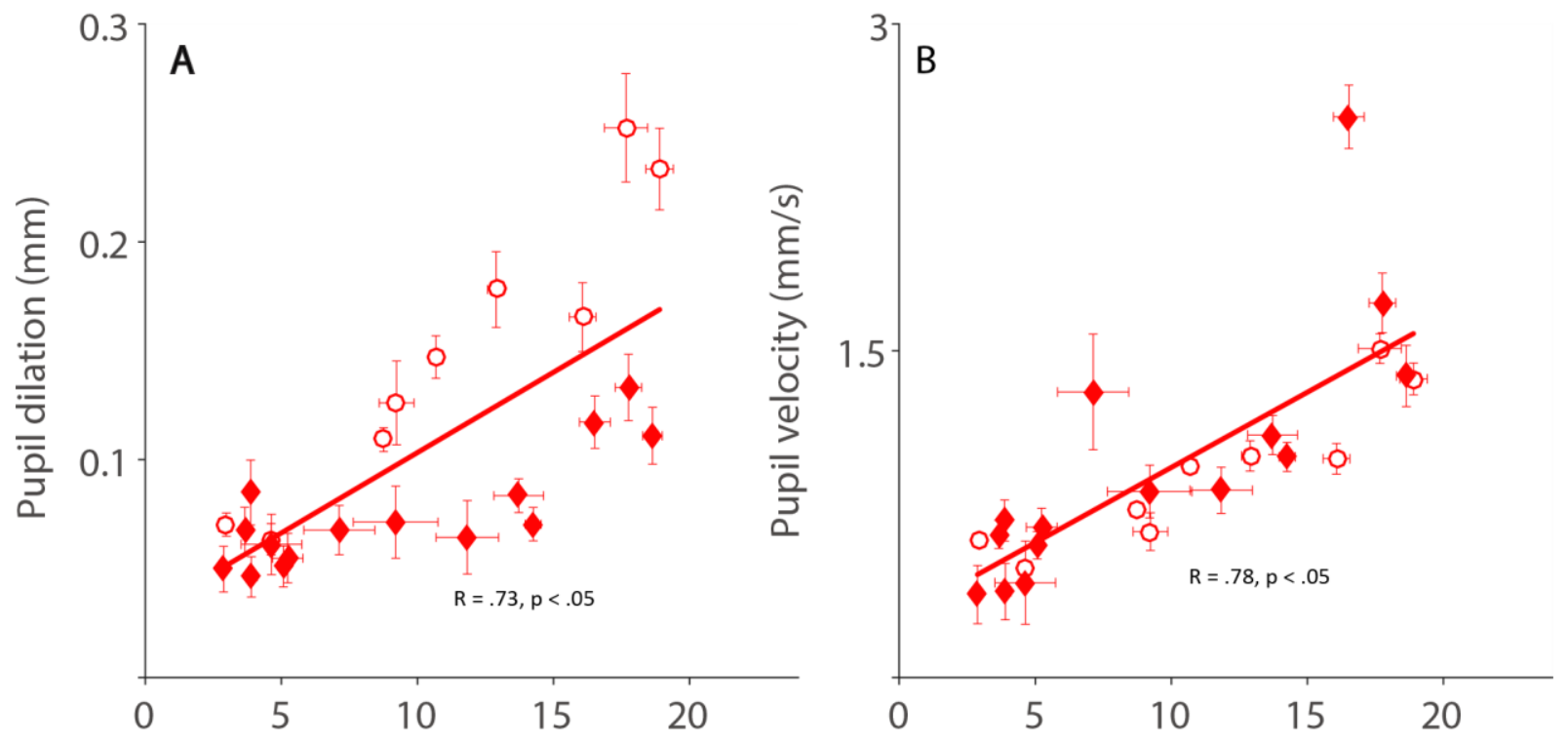

Eccentricity of response field (deg) magnitude and $(\mathbf{B})$ velocity $(\mathrm{n}=24)$. Lines indicate the regression line. Monkey A: $\diamond$, Monkey B: ○. 
Modulation of pupil but not saccade responses by background luminance

The major function of changes in pupil size is to regulate the amount of light projected onto the retina ${ }^{29,30}$. However, whether global luminance signals play a role in modulating the SC is

267 yet to be explored. Varying background luminance changes the balance of activity in the pupil

268 control pathway ${ }^{29}$. The pupil responses evoked by the SCi should thus be affected. More

269 importantly, if the SCi is also involved in luminance encoding as suggested by the results of

270 Hannibal et al. ${ }^{31}$, then varying background luminance should also modulate saccade responses

271 evoked by SCi microstimulation. To investigate the influences of background luminance on

272 pupil and saccade responses evoked by SCi microstimulation, monkeys performed the same

273 fixation task described above, However, this time background luminance was varied, and

274 microstimulation parameters were fixed for each recording site (stimulation duration: $100 \mathrm{~ms}$;

275 stimulation frequency range: $240-300 \mathrm{~Hz}$ ). Changes in background luminance systematically

276 changed pupil diameter, as shown in Fig. 4 for an example stimulation site. Consistent with the

277 literature ${ }^{29}$, increasing background luminance systematically reduced baseline pupil diameter

278 with mean value of $3.07,2.47,2.2,1.79,1.56 \mathrm{~mm}$ in the $2.5,5,10,20,40 \mathrm{~cd} / \mathrm{m}^{2}$ background

279 conditions, respectively (Fig. 4A; differences were all statistically significant between any of two

280 conditions according to bootstrapping, see Materials and Methods). Importantly, pupil responses

281 evoked by SCi microstimulation were also systematically modulated by background luminance

282 levels (Fig. 4B). Increasing background luminance produced lower pupil velocities (Fig. 4C, low

283 to high background luminance: mean value: $1.42,1.26,1.07,0.66,0.85 \mathrm{~mm} / \mathrm{s}$ ) and less dilation

284 (Fig. 4D, low to high background luminance: mean value: 0.38, 0.35, 0.25, 0.16, 0.16 mm). In

285 contrast, saccade responses were not systematically modulated by background luminance, with 

although some comparisons were statistically significant.
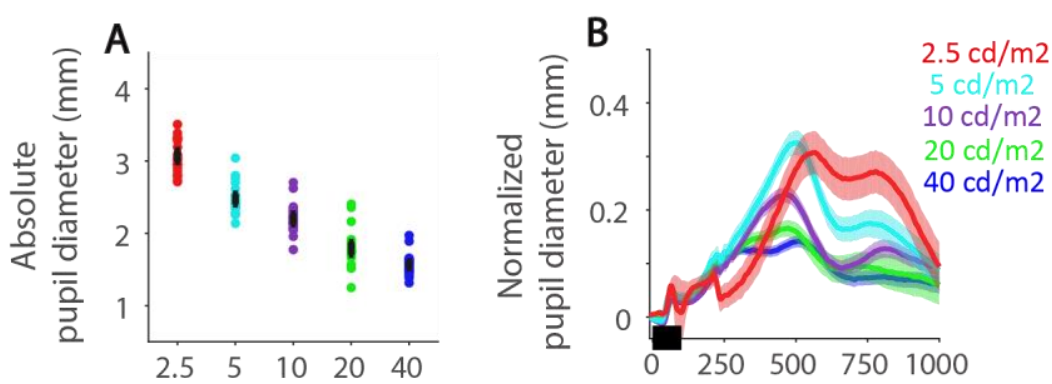

Background luminance $(\mathrm{cd} / \mathrm{m} 2)$ Time from microstimulation (ms)
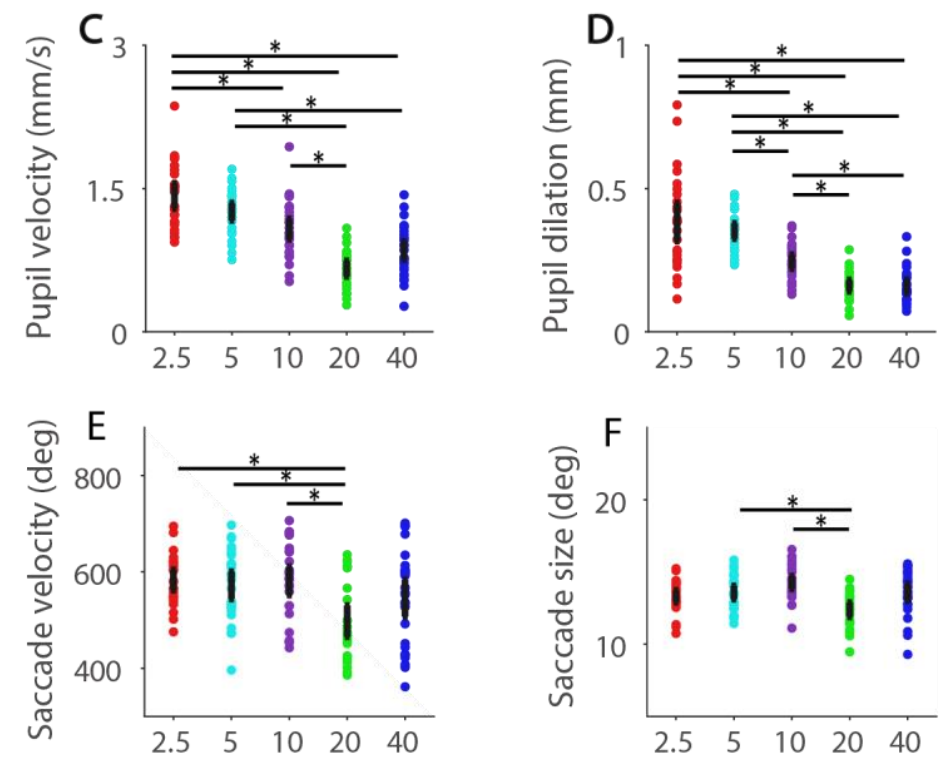

Background luminance $(\mathrm{cd} / \mathrm{m} 2)$

Figure 4. Figure 4. Examples showing background luminance effects on saccade and example site. (B) Pupil responses after microstimulation in five background luminance conditions. (C-D) Pupil responses after microstimulation in different background luminance conditions with respect to (C) velocity and (D) magnitude. (E-F) Saccade responses after microstimulation in different background luminance conditions with respect to (E) velocity and (F) magnitude. In A,C-F, the thick black and gray circles 
represent an average and individual trial values of measurements. In $\mathbf{B}$, the black bar on

$\mathrm{X}$-axis indicates the time line of microstimulation. The shaded colored regions surrounding the pupil response or error-bar represent $\pm 95 \%$ confidence interval for different conditions derived from the bootstrap analysis. $\mathrm{n}$ : number of sites. *: differences were statistically significant according to bootstrapping.

Figure 5 summarizes the effects of SCi microstimulation on saccade and pupil responses in different background conditions $\left(2.5,20,40 \mathrm{~cd} / \mathrm{m}^{2}\right)$ collapsed across all stimulation sites tested with luminance changes from 2 monkeys $(\mathrm{N}=16)$ (note that this was minimal data set for all possible sites and some sites, e.g. Fig 4, had more than 3 luminance values). Pupil diameter (Fig. 5A, sac: $\mathrm{F}(2,30)=1417.86, p<0.001 ;$ Bonferroni-corrected comparisons: all comparisons, $p<$ 0.05) prior to microstimulation was clearly modulated by background luminance, with absolute pupil sizes $2.45,1.35$, and $1.13 \mathrm{~mm}$ for $2.5,20,40 \mathrm{~cd} / \mathrm{m}^{2}$, respectively. Figure $5 \mathrm{~B}$ shows the

310 normalized pupil diameter produced by SCi microstimulation for three background conditions

311 (stimulation versus no-stimulation), revealing larger responses for lower luminance conditions.

312 To quantify these orienting responses, we normalized these responses (see Materials and

313 Methods). As predicted, evoked pupil responses were systematically modulated by background

314 luminance (Fig. 5C: velocity: $\mathrm{F}(2,30)=44.163, p<0.001$, all comparisons $p<0.01$; Fig. 5D:

315 magnitude, $\mathrm{F}(2,30)=221.934, p<0.001$, comparisons between $2.5 \mathrm{~cd} / \mathrm{m}^{2}$ and others, $\left.p<0.05\right)$,

316 with larger pupil dilation evoked with lower background luminance. In contrast, the probability

317 of evoked saccades was not influenced by different background luminance $\left(2,5,20,40 \mathrm{~cd} / \mathrm{m}^{2}\right.$ :

$31894,92,91 \%, \mathrm{~F}(2,30)=0.538, p=0.59)$. Moreover, neither the velocity (Fig. 5E: $\mathrm{F}(2,30)=$

$3191.486, p=0.24$, all comparisons, $p>0.37$ ) nor magnitude (Fig. 5F: $\mathrm{F}(2,30)=0.999, p=0.38$, all 
comparisons, $p>0.5$ ) of the saccades were modulated by background luminance levels. These

321 results characterize the influence of luminance signals on pupil, but not, saccade responses

322 evoked by SCi microstimulation.
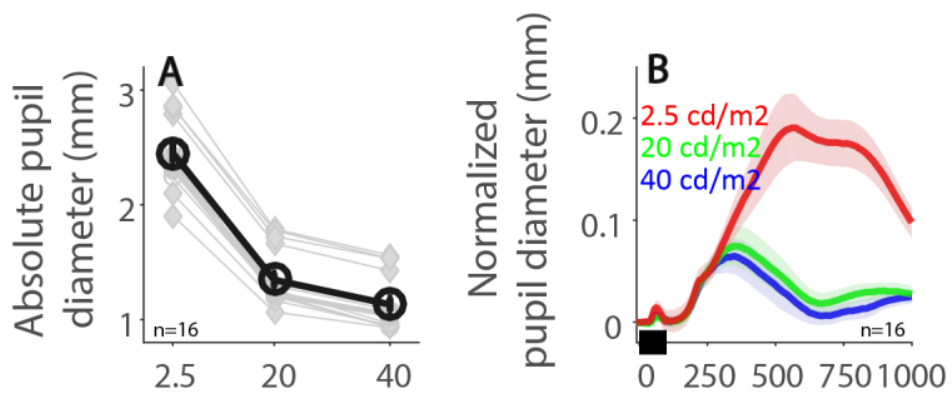

Background luminance (cd/m2) Time from microstimulation (ms)
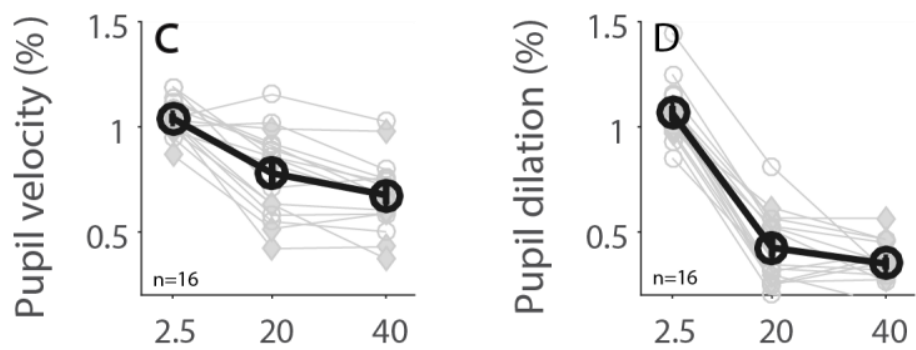
across monkeys and stimulation sites $(n=16)$. (B) Pupil responses after microstimulation for three background luminance conditions. (C-D) Pupil responses after microstimulation 
in (E) velocity and (F) magnitude. In A,C-F, the thick black and gray circles represent an average and individual site values of measurements. In $\mathbf{B}$, the black bar on X-axis indicates the time line of microstimulation. The shaded colored regions surrounding the

\section{Discussion} pupil response represent \pm standard error range for different conditions. n: number of

The goal of this study was to understand the role of the SCi in the coordination between saccades and pupil dilation during the orienting response, and to determine whether background

339 luminance interacts with these evoked responses (Fig. 1A). Varying stimulation frequency

340 systematically modulated the evoked saccade as well as the pupil responses, with larger saccade

341 and pupil responses observed for higher stimulation frequencies. Trial-by-trial correlation was

342 also observed: trials with larger saccade responses had larger pupil responses. Furthermore,

343 microstimulation of SCi sites, which produced larger amplitude saccades, also evoked larger

344 pupil dilation. These results together demonstrate saccade and pupil movements are coordinated

345 through the SCi, likely via a shared common output signal derived from the SCi. However,

346 varying background luminance only influenced evoked pupil responses, implying that luminance

347 information was not processed directly in the SCi, although the SC does receive direct retinal

348 projections $^{31}$.

Circuits for saccade and pupil control by the superior colliculus

The SCi integrates sensory, motor, and cognitive signals from various cortical and

352 subcortical areas and projects directly to the premotor brainstem circuitry to initiate the orienting 
353 response ${ }^{10,12}$. Although the role of the SCi in the control of saccade and pupil responses has

354 been developed, it has yet to be determined whether the same SCi efferent projection drives both

355 saccade and pupil responses (the common drive hypothesis), or alternatively, that saccade and

356 pupil responses are mediated by different sets of axons emerging from the SCi. The connections

357 between neurons in the SCi and brainstem reticular formation for control of saccades is well-

358 established ${ }^{44-46}$. The central mesencephalic reticular formation (cMRF) is one of the collicular

359 targets involved in horizontal saccade control ${ }^{47}$, and research using intra-axonal recording and

360 horseradish peroxidase injection has revealed that saccade-related long-lead burst neurons in the

361 SCi with axon collaterals terminating in the cMRF also commonly project to the pontine

362 premotor centers for saccade control ${ }^{48,49}$. Moreover, it has recently shown that cMRF also

363 projects directly to the Edinger-Westphal nucleus ${ }^{50}$, a critical structure in the pupil control

364 circuit ${ }^{10,12}$. In addition, the superior colliculus projects directly, and indirectly via the cMRF, to

365 the medullary reticular formation ${ }^{51}$, and so may influence preganglionic sympathetic

366 motoneurons via this route. However, connections to the motoneurons controlling dilation via

367 these routes remain to be demonstrated. These results and trial-by-trial correlations together

368 suggest that the same efferent projection derived from the SCi could possibly drive both saccade

369 and pupil premotor circuits to produce coordinated saccade and pupil responses. We found that

370 microstimulation of the SCi produced trial-by-trial correlations between saccade and pupil

371 responses, suggesting that common output signals derived from the SCi drive both down-stream

372 premotor circuits. Although trial-by-trial correlation between saccadic and pupil responses could

373 theoretically be explained by assuming different SCi efferents are activated via current

374 spreading, current spread, anatomical data on SCi efferents supports the common drive

375 hypothesis. 
Monkeys returned their eyes back to the central FP (return saccade) after a saccade evoked by SCi microstimulation. The properties of the return saccade are very similar to the evoked saccade, albeit traveling in the opposite direction. Moreover, pupil size should also be modulated

379 in a manner that correlates with the return saccade. Due to the speed of these two saccades, it 380 appears that the pupil effects were combined. Certainly, robust trial-by-trial correlations were 381 still observed even though we only compared dilation to the properties of the stimulus evoked 382 saccades. Future studies are thus needed to explore how a sequence of saccades might modulate 383 pupil response dyanamics.

Different regions in the SC motor map project disproportionally to omnipause neurons and excitatory/inhibitory burst neurons to trigger saccades with different amplitudes ${ }^{41-43,52}$. Although unidentified, this pattern of anatomical relationship may also be present in the SCi projections to

387 the pupil control circuit; that is, regions in the SCi map that code for larger amplitude saccades

388 (i.e., caudate SC) also code larger pupil dilation, yielding more and/or stronger projections in the caudal SC to the pupil control circuit. Our results support this idea: SC sites that evoked larger

390 size saccades also induced larger pupil dilation. Future research is required to explore the 391 anatomical relationship between different regions in the SC and its connections to the pupil 392 control circuit.

Role of other brain areas in pupil control

Pupil size is also modulated by other structures in the brain that possibly contribute to 396 correlations observed here between saccade and pupil responses ${ }^{53,54}$. Mounting evidance has 397 demonstrated that pupil size covaries with neural activity of the locus coeruleus (LC) in behaving 398 monkeys ${ }^{20,22,55}$, and that LC neurons also discharge phasically to task-relevant sensory stimuli 
56-61. Furthermore, the cholinergic system also correlates with pupil responses ${ }^{21}$, and could also

400 modulate saccade responses through changing cholinergic activity within the SC via input from

401 the pedunculopontine tegmental nucleus (PPTN) ${ }^{62-64}$. Pupil dilation is also evoked by

402 subthreshold microstimulation of the frontal eye fields (FEF) ${ }^{19,65}$, a structure involved in

403 attention and gaze shifts ${ }^{66}$, although these studies focused mainly on pupil dynamics, instead of

404 the coordination between saccade and pupil responses. Therefore, it is possible that

405 abovementioned structures are also involved in observed correlations between pupil and saccade

406 responses. Furthermore, the SCi receives inputs from the LC, FEF as well as cholinergic

407 projections from the PPTN ${ }^{15-18}$. Therefore, it is likely that the signals from LC, FEF, and PPTN

408 are integrated in the SCi to drive coordinated saccade and pupil responses. This idea can also

409 explain why pupil responses are more reliably modulated by saccade responses than by

410 stimulation frequency because the saccade response is the summation of SC inputs (e.g., LC,

411 FEF, cholinergic) and stimulation frequency.

413 Global luminance effects on evoked saccades and pupil dilation and functional role of its

\section{4 coordination}

415 The SC receives retinal projections, including ipRGCs projections to both the SCs and SCi

$416{ }^{31}$, which are particularly important to luminance encoding and the pupillary light reflex ${ }^{32-35}$.

417 However, it is unclear whether these luminance signals serve a role in signal processing in the

418 SCi. Varying background luminance changes pupil size by regulating the activity balance

419 between the sympathetic and parasympathetic pathways, with greater activation in the

420 sympathetic system, but smaller activation in the parasympathetic system, at lower levels of

421 luminance ${ }^{29,67}$. Here, we found that lowering background luminance systematically increased 
422 the degree of pupil dilation evoked by SCi microstimulation, but it did not change the evoked

423 saccade responses. This suggests an independent relationship between the SCi and luminance

424 signal processing. Notably, although the global luminance did not modulate saccades evoked by

425 microstimulation, it may influence saccades that are heavily associated with visual processes

426 such as visually-guided saccades, so, future work is required to examine this question.

427 Pupil dilation can be mediated through inhibiting the parasympathetic system ${ }^{68}$ and/or

428 activating the sympathetic system ${ }^{29,67}$. According to the modulation of the balanced activity

429 between the sympathetic and parasympathetic pathways by background luminance, pupil dilation

430 should be mediated mainly by the excitation of the sympathetic pathway under lower luminance

431 conditions, but by the inhibition of the parasympathetic pathway under higher luminance

432 conditions. Because dilation here was greater in lower global luminance, such dilation was likely

433 due to sympathetically-driven dilator pupillae contraction which is reduced by antagonism from

434 increased sphincter pupillae contraction at higher luminance levels.

435 Larger pupil dilation observed in lower luminance conditions also leads to an intriguing

436 question because pupil dilation should be larger under higher luminance conditions according to

437 the mechanical limit of pupil size. The fact that larger pupil dilations were produced by SCi

438 activation when luminance was lower is intriguing because pupil dilation should theoretically be

439 larger under low light conditions where the pupil is near the mechanical limit of pupil size. Our

440 finding the opposite effect may reflect the functional role of orienting pupil dilation. It has been

441 argured that pupil dilation evoked by salient stimuli serves to slightly increase visual sensitivity

$442{ }^{69}$, so the size of evoked pupil dilation should be larger under lower luminance conditions

443 because it is particularly needed to increase visual sensitivity under these conditions.

444 Consistently, the observed pupil dilation produced by SCi stimulation was larger under lower 
445 background luminance conditions, which is consistent with our previous results with

446 subthreshold microstimulation ${ }^{4}$. More interestingly, saccade and pupil responses evoked by the

$447 \mathrm{SCi}$, although different in time course, are coordinated. Does the coordination between saccade

448 and pupil responses serve any functional role for visual processing? Although the current study

449 cannot address this question, a potential benefit may be having a larger pupil size after

450 orientation, i.e., saccades, resulting in efficient processing of a selected target at the beginning of

451 the foveation. Future research is needed to directly examine the functional role of pupil dilation

452 evoked by salient stimuli and mediated by the SC for visual processing.

\section{Conclusion}

455 The SCi, a hub of sensory and motor processing, integrates sensory and cognitive signals to 456 coordinate the orienting response that includes eye movements and pupil size changes ${ }^{10,12}$. Here, 457 we demonstrated saccades and pupil dilation evoked by SCi microstimulation were highly 458 correlated, and SCi site-specific effects were observed in both saccade and pupil responses, 459 together supporting the common drive hypothesis, and arguing that the same efferent projection 460 from the SCi drives both saccade and pupil responses. Moreover, varying background luminance 461 only modulated evoked pupil, but not saccade, responses, implying the functional independence 462 of the SCi and luminance signals. Orienting responses are thought to work together to optimize 463 the body for whatever action is required ${ }^{69}$. Investigation of the various orienting components 464 simultaneously is thus necessary to understand how they are coordinated to optimize 465 performance. 
Animal experimental setup. Experiments were performed on two male rhesus monkeys

469 (Macaca mulatta; 10 and $11 \mathrm{~kg}$ ). The protocols used in this study were approved by Queen's

470 University Animal Care Committee in accordance with the Canadian Council on Animal Care

471 policies for the use of laboratory animals. The methods of surgical procedures, techniques for

472 extracellular neuronal recording, and data collection have been described in detail previously ${ }^{70}$.

473 Eye position and pupil size were measured by a video-based eye tracker (Eyelink-1000, SR

474 Research, Osgoode, ON, Canada) at a rate of $1000 \mathrm{~Hz}$ with monocular recording (right pupil).

475 Pupil area values recorded from the eye tracker were converted to pupil diameter (see details in ${ }^{5}$.

476 Stimulus presentation and data acquisition were controlled by a UNIX based real-time data

477 control system (REX) (Hays et al., 1982). Spikes, eye position, and pupil diameter were recorded

478 in a multichannel data acquisition system (Plexon). Stimuli were presented on a CRT monitor at

479 a screen resolution of $1024 \times 768$ pixels (75Hz non-interlaced), subtending a viewing angle of 54

$480 \quad x 44 \mathrm{deg}$.

$481 \quad$ Procedure, SC recording, and stimulation. Monkeys were seated in a primate chair with

482 their heads restrained facing the video monitor. We lowered tungsten microelectrodes

483 (impedance: 0.1-1 M $\Omega$, Frederick Haer) to locate the SC. We mapped the visual response fields

484 of the SC using a visual mapping task ${ }^{36}$, and identified the depth of the SCi (intermediate layers

485 of the superior colliculus) using a delayed saccade task. Once the SCi was localized, it was

486 microstimulated $(250-300 \mathrm{~Hz}$ pulse train for $100 \mathrm{~ms}$ with alternating $0.3 \mathrm{~ms}$ anode plus $0.3 \mathrm{~ms}$

487 cathode pulses), and threshold for saccades was determined when the stimulation current in the

488 SCi evoked saccades $50 \%$ of the time (range: 5-50 $\mu \mathrm{A}$ ). The optimal locations of the response

489 fields of SCi neurons were in close agreement with the vector of eye movement elicited with

490 suprathreshold SCi stimulation, and ranged between $3^{\circ}$ and $20^{\circ}$ eccentricity. 
Experimental task. The monkeys were trained to perform a simple fixation task. They had

492 to maintain gaze within $1.5^{\circ}$ of a central fixation point $\left(\mathrm{FP}, 0.5^{\circ}\right.$ diameter; $20 \mathrm{~cd} / \mathrm{m}^{2}$, isoluminant

493 color of the background) at the center of the screen on a gray background $\left(20 \mathrm{~cd} / \mathrm{m}^{2}\right)$ for a few

494 seconds to obtain a liquid reward. After the monkey maintained fixation for 1-1.5 s, a train of

495 stimulation pulses was delivered on $50 \%$ of the trials, and the monkeys had to maintain fixation

496 for another 1.5-2 s, regardless of microstimulation. Saccades were often evoked after electrical

497 stimulation (latencies usually less than $100 \mathrm{~ms}$ ). Monkeys had to move their eyes back to the FP

498 within $500 \mathrm{~ms}$ after microstimulation, and both monkeys usually moved their eyes back to the FP

499 within $300 \mathrm{~ms}$ after microstimulation.

Two microstimulation experiments were conducted. In the first experiment, stimulation

501 frequency was manipulated (100 ms stimulation train duration). After determining the saccade

502 threshold current, usually at $300 \mathrm{~Hz}$ stimulation, we systematically varied the frequency of

503 stimulation, ranging from 150 to $300 \mathrm{~Hz}$, and used $150 \%$ of the saccade threshold current to

504 regularly evoke saccades, particularly under high frequency stimulation. At each stimulation site,

505 we microstimulated at 3-8 different frequency levels. The order of frequency levels across blocks

506 was varied across days. Microstimulation was delivered to 24 sites ( 9 and 15 in monkeys A and

507 B, respectively).

508 In the second experiment, the level of global luminance was manipulated by changing the

509 background luminance level of the computer screen. We thus refer to background lumiance of

510 the screen as global luminance. Stimulation levels were fixed across various background

511 luminance conditions (> $250 \mathrm{~Hz}$ stimulation frequency and $100 \mathrm{~ms}$ stimulation duration). The

512 monkey had to maintain gaze within $1.5^{\circ}$ of a central FP $\left(0.5^{\circ}\right.$ diameter; isoluminant color

513 difference from background) at the center of the screen with background luminance of 2.5, 20, or 
$51440 \mathrm{~cd} / \mathrm{m}^{2}$. The order of luminance levels across blocks was varied across days. Microstimulation

515 was delivered to 16 sites (6 and 10 in monkeys A and B, respectively) at 3-5 background levels

$516\left(2.5,5,10,20,40 \mathrm{~cd} / \mathrm{m}^{2}\right)$. There were at least 20 correct trials in all conditions.

517 Data analysis. Trials with blinks or an eye position deviation of more than $1.5^{\circ}$ from the

518 central FP or with the detected saccades $\left(>2^{\circ}\right)$ during the required period of central fixation were

519 excluded from analysis except for microstimulation-related (evoked and return) saccades. The

520 recorded pupil size depends on the subject's gaze angle in a video-based eye-tracker so that

521 pupil-size data can be distorted by eye movements and eccentric eye positions. Because of

522 applying suprathreshold microstimulation, saccades were often evoked after stimulation and eye

523 position returned to center within $300 \mathrm{~ms}$. To maintain an accurate measure of the pupil, we

524 mainly used pupil peak dilation and velocity measures because they regularly occurred after 300

$525 \mathrm{~ms}$ of microstimulation, and at this time the eyes had returned to the fixation point, so that the

526 pupil could be accurately measured. The difference in the efferent delays of the saccade and

527 pupillary responses are primarily caused by the different muscle types being innervated: smooth

528 muscle controlling the pupil and extraocular fast twitch skeletal fibers for the saccades.

529 Nevertheless, the pupil response is temporally dissociated from the saccade response (see Fig.

530 1B for an example).

531 Because the pupil response is consensual ${ }^{4,5}$, only pupil diameter of the right eye was

532 recorded for data analysis. Following the procedures of baseline-correction used previously ${ }^{4,6}$,

533 original pupil diameter values were subtracted from the baseline pupil diameter value determined

534 by averaging pupil size from $200 \mathrm{~ms}$ before to the onset of microstimulation for each trial. To

535 normalize pupil and saccade measurements across conditions for population analyses (frequency,

536 background luminance), all measured values were divided by the median value of the highest 
537 frequency condition (lowest luminance level for the other experiments). To be included for

538 condition-based analyses, each condition (frequency/background luminance) had to have more

539 than 15 remaining trials, except where indicated. Evoked saccade reaction time (SRT) was

540 defined as the time after microstimulation to the first saccade away from central fixation that

541 exceeded $30 \%$ s. The highest three frequency conditions were selected for some analyses to

542 examine the relationship between saccade and pupil responses because there were more

543 numerous trials with evoked saccades in the higher frequency conditions.

544 Results are shown as mean \pm SEM. We performed correlational analyses and a two-tailed

545 student $t$ test except where indicated. Bayesian $t$ tests, where appropriate, were also performed to

546 inform statistical significance for pairwise comparisons, with a scale factor $\mathrm{r}=0.707^{71}$.

547 Moreover, Cohen's $d$, where appropriate, was calculated to estimate effect size ${ }^{72}$. In the

548 background luminance experiment, we used a bootstrap method to inform the statistical

549 significance of the comparison by performing a random sampling of pupil values derived from

550 each recording trial with 1000 repetitions ${ }^{6}$. This resulted in a normally distributed cluster of

551 points centered on the mean of selected pupil values (clusters not shown, normal distribution was

552 verified by the Kolmogorov-Smirnov test). Statistical tests were performed using JASP Team

553 (2019) and MATLAB (The MathWorks Inc., Natrick, MA, USA). 


\section{References}

556 1. Sokolov, E. N. Higher Nervous Functions: The Orienting Reflex. Annu. Rev. Physiol. 25,

$557 \quad 545-580(1963)$.

558 2. Akert, K. Der visuelle Greifreflex. Helv. Physiol. Pharmacol. Acta 7, 112-134 (1949).

559 3. Hess, W. R., Buergi, S. \& Bucher, V. Motorische Funktionen des Tektal - und

560 Tegmentalgebietes (motor functions of tectal and tegmental areas). Monatsschr. Psychiatr.

$561 \quad$ Neurol. 112, 1-52 (1946).

562 4. Wang, C.-A., Boehnke, S. E., White, B. J. \& Munoz, D. P. Microstimulation of the

563 monkey superior colliculus induces pupil dilation without evoking saccades. J. Neurosci.

$564 \quad 32,3629-3636(2012)$.

565 5. Wang, C.-A. \& Munoz, D. P. Modulation of stimulus contrast on the human pupil

566 orienting response. Eur. J. Neurosci. 40, 2822-2832 (2014).

567 6. Wang, C.-A., Boehnke, S. E., Itti, L. \& Munoz, D. P. Transient pupil response is

568 modulated by contrast-based saliency. J.Neurosci. 34(2), 408-417 (2014).

569 7. Wang, C.-A., Blohm, G., Huang, J., Boehnke, S. E. \& Munoz, D. P. Multisensory

570 integration in orienting behavior: Pupil size, microsaccades, and saccades. Biol. Psychol.

$571 \quad \mathbf{1 2 9}, 36-44(2017)$.

572 8. Gandhi, N. J. \& Katnani, H. A. Motor functions of the superior colliculus. Annu. Rev.

$573 \quad$ Neurosci. 34, 205-231 (2011).

574 9. Krauzlis, R. J., Lovejoy, L. P. \& Zenon, A. Superior colliculus and visual spatial attention.

575 Annu. Rev. Neurosci. 36, 165-182 (2013).

576 10. Corneil, B. D. \& Munoz, D. P. Overt Responses during Covert Orienting. Neuron 82,

$577 \quad 1230-1243(2014)$. 
578 11. Hall, W. C. \& Moschovakis, A. The Superior Colliculus: New Approaches for Studying

579 Sensorimotor Integration. Methods \& New Frontiers in Neuroscience. (2003).

580 12. Wang, C.-A. \& Munoz, D. P. A circuit for pupil orienting responses: implications for cognitive modulation of pupil size. Curr. Opin. Neurobiol. 33, 134-140 (2015).

582 13. Eckstein, M. K., Guerra-Carrillo, B., Miller Singley, A. T. \& Bunge, S. A. Beyond eye gaze: What else can eyetracking reveal about cognition and cognitive development? Dev. Cogn. Neurosci. 25, 69-91 (2017).

14. White, B. J. \& Munoz, D. P. The Superior colliculus. in Oxford Handbook of Eye Movements (ed. Liversedge Gilchrist, I. and Everling, S., S.) vol. 2011 195-213 (Oxford University Press, 2011).

15. Edwards, S. B. Autoradiographic studies of the projections of the midbrain reticular formation: descending projections of nucleus cuneiformis. J. Comp. Neurol. 161, 341-358 (1975).

16. Wurtz, R. H. \& Albano, J. E. Visual-motor function of the primate superior colliculus . Annu. Rev. Neurosci. 3, 189-226 (1980).

593 17. Beninato, M. \& Spencer, R. F. A cholinergic projection to the rat superior colliculus

18. Graybiel, A. M. A stereometric pattern of distribution of acetylthiocholinesterase in the deep layers of the superior colliculus. Nature 272, 539-541 (1978).

598 19. Lehmann, S. J. \& Corneil, B. D. Transient Pupil Dilation after Subsaccadic Microstimulation of Primate Frontal Eye Fields. J. Neurosci. 36, 3765-76 (2016).

600 20. Aston-Jones, G. \& Cohen, J. D. An integrative theory of locus coeruleus-norepinephrine 
function: adaptive gain and optimal performance . Annu. Rev. Neurosci. 28, 403-450

602 (2005).

603 21. Reimer, J. et al. Pupil fluctuations track rapid changes in adrenergic and cholinergic activity in cortex. Nat. Commun. 7, 13289 (2016).

22. Joshi, S., Li, Y., Kalwani, R. M. \& Gold, J. I. Relationships between Pupil Diameter and Neuronal Activity in the Locus Coeruleus, Colliculi, and Cingulate Cortex. Neuron 89, 221-34 (2016).

23. Nelson, A. \& Mooney, R. The Basal Forebrain and Motor Cortex Provide Convergent yet Distinct Movement-Related Inputs to the Auditory Cortex. Neuron 90, 635-648 (2016).

24. Sparks, D. L. Translation of sensory signals into commands for control of saccadic eye movements: role of primate superior colliculus . Physiol. Rev. 66, 118-171 (1986).

25. Robinson, D. A. Eye movements evoked by collicular stimulation in the alert monkey . Vision Res. 12, 1795-1808 (1972).

614 26. Stanford, T. R., Freedman, E. G. \& Sparks, D. L. Site and parameters of microstimulation: evidence for independent effects on the properties of saccades evoked from the primate superior colliculus. J. Neurophysiol. 76, 3360-3381 (1996).

617 27. Laughlin, S. B. Retinal information capacity and the function of the pupil. Ophthalmic Physiol. Opt. 12, 161-164 (1992).

619 28. Woodhouse, J. M. The effect of pupil size on grating detection at various contrast levels. Vision Res. 15, 645-648 (1975).

621 29. Loewenfeld, I. E. The pupil: anatomy, physiology, and clinical applications. (Boston: Butterworth- Heinemann., 1999).

623 30. McDougal, D. H. \& Gamlin, P. D. Autonomic control of the eye. Compr. Physiol. 5, 439- 
$73(2015)$.

625 31. Hannibal, J. et al. Central projections of intrinsically photosensitive retinal ganglion cells 626 in the macaque monkey. J Comp Neurol 522, 2231-2248 (2014).

627 32. Do, M. T. H. \& Yau, K.-W. Intrinsically photosensitive retinal ganglion cells. Physiol. Rev. 90, 1547-81 (2010).

629 33. Gamlin, P. D. R. et al. Human and macaque pupil responses driven by melanopsincontaining retinal ganglion cells . Vision Res. 47, 946-954 (2007).

631 34. Münch, M. \& Kawasaki, A. Intrinsically photosensitive retinal ganglion cells: classification, function and clinical implications. Curr. Opin. Neurol. 26, 45-51 (2013).

633 35. Dacey, D. M. et al. Melanopsin-expressing ganglion cells in primate retina signal colour and irradiance and project to the LGN . Nature 433, 749-754 (2005).

635 36. Marino, R. A. et al. Linking visual response properties in the superior colliculus to

37. Scudder, C. A., Kaneko, C. R. \& Fuchs, A. F. The brainstem burst generator for saccadic eye movements: A modern synthesis. Exp. Brain Res. 142, 439-462 (2002).

639 38. Ferrier, D. The functions of the brain. (G P Putnam's Sons, 1876). doi:10.1037/12860$640 \quad 000$.

641 39. Beatty, J. Task-evoked pupillary responses, processing load, and the structure of processing resources . Psychol. Bull. 91, 276-292 (1982).

643 40. van der Wel, P. \& van Steenbergen, H. Pupil dilation as an index of effort in cognitive 644 control tasks: A review. Psychon. Bull. Rev. 25, 2005-2015 (2018).

645 41. Büttner-Ennever, J. A., Horn, A. K., Henn, V. \& Cohen, B. Projections from the superior 646 colliculus motor map to omnipause neurons in monkey. J. Comp. Neurol. 413, 55-67 
(1999).

648 42. Moschovakis, A. K., Dalezios, Y., Petit, J. \& Grantyn, A. A. New mechanism that accounts for position sensitivity of saccades evoked in response to stimulation of superior

43. Moschovakis, A. K. et al. An anatomical substrate for the spatiotemporal transformation. J. Neurosci. 18, 10219-29 (1998).

44. Sparks, D. L. The brainstem control of saccadic eye movements. Nat. Rev. Neurosci. 3, 952-964 (2002).

45. Horn, A. K. E. The reticular formation. in Progress in brain research vol. 151 127-155 (2006).

46. May, P. J. The mammalian superior colliculus: laminar structure and connections . Prog. Brain Res. 151, 321-378 (2006).

47. Cohen, B. \& Büttner-Ennever, J. A. Projections from the superior colliculus to a region of the central mesencephalic reticular formation (cMRF) associated with horizontal saccadic eye movements. Exp. brain Res. 57, 167-76 (1984).

48. Scudder, C. A., Moschovakis, A. K., Karabelas, A. B. \& Highstein, S. M. Anatomy and physiology of saccadic long-lead burst neurons recorded in the alert squirrel monkey. I.

49. Grantyn, A. \& Grantyn, R. Axonal patterns and sites of termination of cat superior colliculus neurons projecting in the tecto-bulbo-spinal tract. Exp. brain Res. 46, 243-56 (1982).

50. May, P. J., Warren, S., Bohlen, M. O., Barnerssoi, M. \& Horn, A. K. E. A central mesencephalic reticular formation projection to the Edinger-Westphal nuclei. Brain 
Struct. Funct. 221, 4073-4089 (2016).

671 51. Perkins, E., Warren, S. \& May, P. J. The Mesencephalic Reticular Formation as a Conduit for Primate Collicular Gaze Control: Tectal Inputs to Neurons Targeting the Spinal Cord and Medulla. Anat. Rec. Adv. Integr. Anat. Evol. Biol. 292, 1162-1181 (2009).

674 52. Gandhi, N. J. \& Keller, E. L. Spatial distribution and discharge characteristics of superior colliculus neurons antidromically activated from the omnipause region in monkey. $J$. Neurophysiol. 78, 2221-2225 (1997).

53. Sara, S. J. \& Bouret, S. Orienting and reorienting: the locus coeruleus mediates cognition through arousal. Neuron 76, 130-141 (2012).

54. Nieuwenhuis, S., De Geus, E. J. \& Aston-Jones, G. The anatomical and functional relationship between the P3 and autonomic components of the orienting response. Psychophysiology 48, 162-175 (2011).

55. Varazzani, C., San-Galli, A., Gilardeau, S. \& Bouret, S. Noradrenaline and Dopamine Neurons in the Reward/Effort Trade-Off: A Direct Electrophysiological Comparison in Behaving Monkeys. J. Neurosci. 35, 7866-7877 (2015).

56. Kalwani, R. M., Joshi, S. \& Gold, J. I. Phasic Activation of Individual Neurons in the Locus Ceruleus/Subceruleus Complex of Monkeys Reflects Rewarded Decisions to Go

57. Swick, D., Pineda, J. A., Schacher, S. \& Foote, S. L. Locus coeruleus neuronal activity in awake monkeys: relationship to auditory P300-like potentials and spontaneous EEG. Exp. brain Res. 101, 86-92 (1994).

58. Grant, S. J., Aston-Jones, G. \& Redmond, D. E. Responses of primate locus coeruleus neurons to simple and complex sensory stimuli. Brain Res. Bull. 21, 401-410 (1988). 
693 59. Rajkowski, J., Kubiak, P. \& Aston-Jones, G. Locus coeruleus activity in monkey: Phasic 694 and tonic changes are associated with altered vigilance. Brain Res. Bull. 35, 607-616 (1994).

60. Rajkowski, J., Majczynski, H., Clayton, E. \& Aston-Jones, G. Activation of monkey locus coeruleus neurons varies with difficulty and performance in a target detection task. $J$. Neurophysiol. 92, 361-371 (2004).

61. Aston-Jones, G., Rajkowski, J., Kubiak, P. \& Alexinsky, T. Locus coeruleus neurons in monkey are selectively activated by attended cues in a vigilance task. J. Neurosci. 14, 4467-4480 (1994).

62. Aizawa, H., Kobayashi, Y., Yamamoto, M. \& Isa, T. Injection of Nicotine Into the Superior Colliculus Facilitates Occurrence of Express Saccades in Monkeys. J. Neurophysiol. 82, 1642-1646 (1999).

63. Li, F., Endo, T. \& Isa, T. Presynaptic muscarinic acetylcholine receptors suppress GABAergic synaptic transmission in the intermediate grey layer of mouse superior colliculus. Eur. J. Neurosci. 20, 2079-2088 (2004).

64. Watanabe, M., Kobayashi, Y., Inoue, Y. \& Isa, T. Effects of Local Nicotinic Activation of the Superior Colliculus on Saccades in Monkeys. J. Neurophysiol. 93, 519-534 (2005).

710 65. Ebitz, R. B. \& Moore, T. Selective Modulation of the Pupil Light Reflex by Microstimulation of Prefrontal Cortex. J. Neurosci. 37, 5008-5018 (2017).

712 66. Thompson, K. G. \& Bichot, N. P. A visual salience map in the primate frontal eye field. Prog. Brain Res. 147, 249-262 (2005).

714 67. Steinhauer, S. R. \& Hakerem, G. The pupillary response in cognitive psychophysiology and schizophrenia. Ann. N. Y. Acad. Sci. 658, 182-204 (1992). 
716 68. Barnerssoi, M., May, P. J. \& Horn, A. K. E. GABAergic innervation of the ciliary

717 ganglion in macaque monkeys - A light and electron microscopic study. J. Comp. Neurol.

$718 \quad 525,1517-1531(2017)$.

719 69. Lynn, R. Attention, arousal and the orientation reaction. (1966).

720 70. Marino, R. A., Rodgers, C. K., Levy, R. \& Munoz, D. P. Spatial relationships of

721 visuomotor transformations in the superior colliculus map . J. Neurophysiol. 100, 2564-

$2576(2008)$.

723 71. Rouder, J. N., Speckman, P. L., Sun, D., Morey, R. D. \& Iverson, G. Bayesian t tests for

724 accepting and rejecting the null hypothesis. Psychon. Bull. Rev. 16, 225-37 (2009).

725 72. Hentschke, H. \& Stüttgen, M. C. Computation of measures of effect size for neuroscience 726 data sets. Eur. J. Neurosci. 34, 1887-1894 (2011). 